\title{
Estado de los parámetros de habla en usuarios con enfermedad pulmonar obstructiva crónica (EPOC), reporte de una serie de casos
}

Joaquín Gay 1,a, Daniela Arredondo ", Sandra Barría ${ }^{1}$, Atalia Catrileo ${ }^{1}$, Evanely Sepúlveda ${ }^{1}$, Carla Figueroa ${ }^{1,2,3, b}$, María Paz Moya ${ }^{1, b}$

RESUMEN

Objetivo: Caracterizar los parámetros de habla en pacientes con enfermedad pulmonar obstructiva crónica (EPOC). Materiales y métodos: Serie de casos prospectiva. En todos los pacientes se realizó una evaluación de los siguientes parámetros del habla: tiempo máximo fonatorio, control motor oral, articulación, lectura, diadococinesias verbales y habla automática. Todos ellos fueron descritos a través de media, mediana y desviación estándar con el software SPSS versión 23.

Resultados: Al estudio ingresaron 20 sujetos ( 7 hombres y 13 mujeres), edad promedio $64,4 \pm 9,1$ años. En cuanto



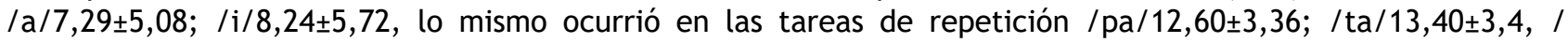
$\mathrm{ka} / 12 \pm 4,11$ y número de palabras por minuto $109 \pm 26,25$.

Conclusiones: Los resultados de este estudio sugieren que los pacientes con EPOC presentan un deterioro en los parámetros del habla, lo que representa una posibilidad de ampliar investigaciones en esta línea y con ello considerar el habla en atención clínica integral.

Palabras clave: Enfermedad pulmonar obstructiva crónica; Fonación; Habla (Fuente: DeCS BIREME).

\section{State of speech parameters in subjects with chronic obstructive pulmonary disease (COPD): a case-series report}

\section{ABSTRACT}

Objective: To characterize the speech parameters in patients with chronic obstructive pulmonary disease (COPD). Materials and methods: A prospective case-series study. All patients underwent an evaluation of the following speech parameters: maximum phonation time, oral-motor control, articulation, reading, verbal diadochokinesia, and automatic speech. The mean, median and standard deviation of these parameters were determined using SPSS software version 23.

Results: Twenty (20) subjects (7 males and 13 females) whose average age was $64.4 \pm 9.1$ years entered the study. The speech parameters showed a reduction in the maximum phonation time (MPT) in /s/ $6.58 \pm 6.76, / \mathrm{a} / 7.29 \pm$ $5.08, / \mathrm{i} / 8.24 \pm 5.72$. The same happened in the repetition tasks: /pa/ $12.60 \pm 3.36, / \mathrm{ta} / 13.40 \pm 3.4, / \mathrm{ka} / 12 \pm$ 4.11 , and the number of words per minute accounted for $109 \pm 26.25$.

Conclusions: The study results suggest that patients with COPD show an impairment in speech parameters. This represents an opportunity to increase research on this topic and thus consider the speech in the comprehensive clinical care.

Keywords: Chronic obstructive pulmonary disease; Phonation; Speech (Source: MeSH NLM).

1. Universidad Autónoma de Chile, Facultad de Ciencias de la Salud, Carrera de Fonoaudiología. Temuco, Chile.

2. Universidad Autónoma de Chile, Centro Investigación Multidisciplinario de La Araucanía (CIMA). Temuco, Chile.

3. Universidad de La Frontera, Programa de Doctorado en Ciencias Médicas. Temuco, Chile.

a. Docente Instructor.

b. Docente Asistente. 


\section{INTRODUCCIÓN}

La OMS proyecta un ascenso, tanto en el número de diagnósticos como en la tasa de mortalidad, para la enfermedad pulmonar obstructiva crónica (EPOC) $(1,2)$ $y$, aunque la producción de habla es alterada por la enfermedad pulmonar, no ha sido incluida en las herramientas actuales de evaluación ${ }^{(3)}$. De lo anterior se desprende la importancia de investigar los alcances de esta patología, para así contribuir en la mejora de la calidad de vida de los pacientes con este diagnóstico, ya que se estima que, conforme la enfermedad va en progreso la funcionalidad de los individuos se va limitando ${ }^{(4)}$.

La enfermedad pulmonar obstructiva crónica (EPOC) se caracteriza por ser un fenómeno inflamatorio de la vía aérea, responsable del aumento de la resistencia al paso del aire y que se acompaña de importantes síntomas sistémicos ${ }^{(4)}$. En la EPOC se encuentra afectada la función ventilatoria, la cual es considerada una función crucial para la producción del habla ${ }^{(5)}$.

Al contrario de lo que ocurre en la espiración normal que es pasiva, en la fonación se genera una espiración activa que produce una corriente de aire favoreciendo la vibración de los pliegues vocales de la laringe en aducción, lo que genera la fonación ${ }^{(4,6)}$. Las tareas fonéticas imponen una demanda respiratoria considerable, tanto en términos de flujo espiratorio como del volumen pulmonar espiratorio (3) , lo que provoca una disminución del tiempo inspiratorio y un aumento en el tiempo espiratorio, por lo que se observa una mayor demanda de flujo espiratorio en tareas de fonación en personas sanas ${ }^{(7)}$. La presión de retracción elástica en el pulmón está determinada principalmente por las fibras elásticas del tejido pulmonar y por la tensión superficial del líquido que recubre el interior de los alvéolos, en la EPOC existe pérdida de retracción elástica del parénquima pulmonar lo que favorece la obstrucción al flujo aéreo ${ }^{(5)}$. En estos pacientes, se observan dificultades, ya que sus flujos son restringidos ${ }^{(8)}$, por lo que se produce una interrupción en la generación de frases, debido a que ellos pueden mantener un índice de flujo solo a volúmenes pulmonares más altos, lo que podría ser explicado por el aumento de la tensión muscular espiratoria progresiva, que promueve un colapso de la vías respiratorias y favorecen la activación de los receptores de las vías respiratorias superiores, para iniciar respuestas aferentes vagales que contribuyen a la disnea ${ }^{(9,10)}$.

En la actualidad resulta necesario conocer el estado en el que se encuentran los parámetros del habla en usuarios con EPOC, a fin de medir la funcionalidad de estos desde el punto de vista comunicativo, ya que estos parámetros (tiempo máximo fonatorio, control motor oral, articulación, lectura, diadococinesias orales y habla automática), corresponden a un pilar crucial en el proceso de transmisión de información de tipo verbal (11). Durante la evaluación clínica del habla, se consideran tareas de movimientos orofaciales (no verbales), repetición de secuencias de sílabas diadococinéticas y prolongación de vocales (paraspeech; tareas de habla simplificada sin significado) y producción de palabras o series de palabras (tareas de habla) ${ }^{(12)}$.

Si bien en la actualidad no se encuentran a nivel nacional investigaciones que relacionen los parámetros de habla con la enfermedad pulmonar obstructiva crónica (EPOC), es ampliamente conocida e investigada la limitación que esta patología provoca en el flujo aéreo de quienes la padecen (2$5,13,14)$. Debido al carácter multidimensional de la enfermedad, su evaluación funcional abarca un amplio espectro de alteraciones, entre las que destacan la obstrucción al flujo aéreo, el desequilibrio de la relación ventilación/perfusión, la limitación de la tolerancia al ejercicio, la afectación de los músculos respiratorios y sus repercusiones sistémicas (14). Oyarzún en 2002, al realizar una búsqueda bibliográfica sobre ¿Qué se investiga en Chile con relación al EPOC? evidencia que los principales tópicos de investigación son entrenamiento físico en EPOC, uso de ventilación mecánica no invasiva y entrenamiento de los músculos inspiratorios (15), pero no se encuentran tópicos que abarquen el aspecto de la comunicación en estos pacientes, lo que representa un vacío en la literatura en torno a cómo el habla no ha sido considerada dentro de alguno de los cuestionarios destinados medir calidad de vida en estos pacientes ${ }^{(16-18)}$. Es por ello que el objetivo de este estudio fue caracterizar los parámetros de habla en pacientes con EPOC.

\section{MATERIALES Y MÉTODOS}

\section{Diseño de investigación}

Se diseñó un estudio de serie de casos prospectivo ${ }^{(19)}$, a través de un muestreo por conveniencia que consideró 20 pacientes diagnosticados de EPOC con clasificación GOLD 1 y GOLD 2 (de su siglas en inglés Global Initiative for Chronic Onstructive Lung Disease), que asistían al CESFAM Conunhueno de la Comuna de Padre las Casa, en el año 2016. Se definieron como criterios de inclusión/exclusión: presencia de EPOC, nacionalidad chilena, hablante nativo del español, rango de edad entre 45 y 80 años, puntuación mayor o igual a 21 puntos en el Test Minimental (MMSE) (20); como criterio de exclusión se consideró presentar patología neurológica y/o cardiovascular.

Los materiales utilizados en este estudio fueron ficha clínica, anamnesis, Mini-Mental State Examination (MMSE) (20) y Protocolo de Evaluación de Parámetros Cuantificables del Habla ${ }^{(21)}$.

Procedimientos de recolección de datos

A los participantes se les realizó una evaluación clínica que 
consideró la ficha del paciente y la anamnesis, luego fueron sometidos a la aplicación del MMSE continuando con la aplicación del Protocolo de Evaluación de Parámetros Cuantificables del Habla ${ }^{(21)}$, que contemplo los siguientes ítems:

1. Tiempo máximo de fonación (TMF): consiste en el tiempo máximo, en segundos, durante el cual el paciente logra mantener de forma ininterrumpida la fonación de los fonemas $/ \mathrm{s} /, \mathrm{/a} / \mathrm{e} / \mathrm{i} /$.

2. Control motor oral y articulación: constituido por la cantidad máxima de repeticiones (ciclos) correctas de las sílabas $/ \mathrm{pa} /, / \mathrm{ta} / \mathrm{y} / \mathrm{ka} /$, por separado, que el paciente realiza en 5 segundos y los ciclos de movimientos orales ejecutados (apertura-cierre mandibular, protrusión-retracción lingual, movimientos linguales laterales, ascenso-descenso lingual, aperturacierre labial y protrusión-retracción lingual) en 5 segundos.

3. Velocidad de lectura: corresponde a la cantidad de palabras producidas en un minuto, obtenidas mediante el cálculo del tiempo en segundos en que el paciente realizó la lectura de un texto de 101 palabras.

4. Diadococinesias orales: consisten en la cantidad de repeticiones correctas de la serie de sílabas /pa-ta-ka/ y palabras (pitúko, petáka, botíka, bodéga, médiko y málaga) realizadas en 5 segundos.

5. Habla automática: correspondiente a la cantidad de segundos en que el paciente realiza la secuencia de contar desde el número 1 al 20.

Previo a la evaluación, se realizó un pilotaje para ver el comportamiento de las pruebas, posterior a ello, cada una de las evaluaciones se realizó bajo condiciones óptimas tanto de espacio, tiempo, iluminación y ruido, para desarrollar de forma correcta la medición de las variables de estudio.

\section{Análisis de los datos}

Para el análisis estadístico los datos fueron introducidos al paquete estadístico IBM SPSS versión 23. Se realizó un análisis exploratorio para conocer la distribución de las variables en estudio, aplicándose la prueba de Shapiro Wilk, según el género. En relación con el análisis descriptivo se obtuvieron medias, medianas y desviaciones estándar.

\section{Consideraciones éticas}

El proyecto respeta los principios declarados en el Tratado de Helsinki y fue aprobado por el comité de ética de la Carrera de Fonoaudiología de la Universidad Autónoma de Chile. Cada uno de los sujetos dio su consentimiento informado previo a la recolección de datos.

\section{RESULTADOS}

Al estudio ingresaron 20 pacientes $(7$ hombres y 13 mujeres), con una edad promedio 64,4 años. En relación a las características generales de los pacientes con EPOC, 8 personas presentaron EPOC por biomasa, 1 persona de tipo genético y 11 personas por hábito tabáquico; en relación al estado cognitivo ninguno obtuvo un rendimiento en la prueba MMSE bajo el puntaje propuesto. (Tabla 1).

Tabla 1. Descriptivos demográficos de los participantes

\begin{tabular}{|c|c|c|c|}
\hline \multicolumn{4}{|l|}{ Género } \\
\hline & & Frecuencia & Porcentaje \\
\hline & Femenino & 13 & 65,0 \\
\hline & Masculino & 7 & 35,0 \\
\hline & Total & 20 & 100,0 \\
\hline \multicolumn{4}{|c|}{ Tipo de EPOC } \\
\hline & & Frecuencia & Porcentaje \\
\hline & Biomasa & 8 & 40,0 \\
\hline & Genético & 1 & 5,0 \\
\hline & Tabáquico & 11 & 55,0 \\
\hline & Total & 20 & 100,0 \\
\hline \multicolumn{4}{|c|}{ Medidas de tendencia central } \\
\hline \multirow{3}{*}{$\begin{array}{l}\text { Edad } \\
\text { Puntaje MMSE }\end{array}$} & $\mathrm{N}$ & $x$ & $\sigma$ \\
\hline & 20 & 64,450 & 9,1679 \\
\hline & 20 & 24,850 & 2,4121 \\
\hline
\end{tabular}


En relación a los parámetros del habla, en el promedio de TMF, fue posible observar una reducción en la duración de la emisión de sonidos sonoros y áfonos, al compararlo con datos referenciales para la población sin EPOC (Tabla 2).

Tabla 2. Descriptivos de los tiempos máximos de fonación de los participantes

\begin{tabular}{cccccccccc} 
Parámetro & \multicolumn{9}{c}{ TMF } \\
& \multicolumn{1}{c}{ Femenino } & \multicolumn{9}{c}{ Masculino } \\
& $x / \sigma$ & Ref & Me & $x / \sigma$ & Ref & Me & $x / \sigma$ & Me \\
\hline Is/ & $5,24 \pm 3,51$ & 17,98 & 3,38 & $9,06 \pm 10,43$ & 21,46 & 6,93 & $6,58 \pm 6,76$ & 3,99 \\
la/ & $6,08 \pm 4,21$ & 15,03 & 4,3 & $7,87 \pm 4,78$ & 16,30 & 4,78 & $7,29 \pm 5,08$ & 6,35 \\
lil & $8,12 \pm 5,44$ & 17,41 & 5,88 & $8,46 \pm 6,50$ & 20,13 & 8,37 & $8,24 \pm 5,72$ & 5,98 \\
\hline
\end{tabular}

TMF: Tiempo máximo fonatorio medido en segundos. Ref: Valores refereciales en población sin patología.

En cuanto a movimientos orales de apertura y cierre mandibular se observó un desempeño más bajo al compararlo con datos referenciales, en el resto de las tareas se observaron mínimas diferencias (Tabla 3).

Tabla 3. Descriptivos de movimientos orales

\begin{tabular}{|c|c|c|c|c|c|c|c|c|}
\hline \multicolumn{9}{|c|}{ Movimientos orales } \\
\hline & \multicolumn{3}{|c|}{ Femenino } & \multicolumn{3}{|c|}{ Masculino } & \multicolumn{2}{|c|}{ Total } \\
\hline & $x / \sigma$ & Ref & Me & $x / \sigma$ & Ref & $M e$ & $x / \sigma$ & Me \\
\hline APCM & $6,77 \pm 2,04$ & 13,13 & 7 & $8,57 \pm 2,22$ & 13,13 & 8 & $7,40 \pm 2,23$ & 7,5 \\
\hline APCL & $5,54 \pm 2,40$ & 14,58 & 6 & $9,43 \pm 3,3$ & 14,58 & 9 & $6,90 \pm 3,27$ & 7 \\
\hline PRLA & $5 \pm 2,27$ & 6,19 & 4 & $7,29 \pm 2,69$ & 6,19 & 8 & $5,80 \pm 2,69$ & 5 \\
\hline PRLE & $5,92 \pm 1,38$ & 6,07 & 6 & $7,71 \pm 4,15$ & 6,07 & 7 & $6,55 \pm 2,72$ & 6 \\
\hline ML & $6,31 \pm 2,72$ & 8,21 & 6 & $7,14 \pm 4,74$ & 8,21 & 6 & $6,60 \pm 3,45$ & 6 \\
\hline MAD & $4,92 \pm 1,49$ & 5,77 & 5 & $4,57 \pm 2,14$ & 5,98 & 4 & $4,80 \pm 1,70$ & 4,5 \\
\hline
\end{tabular}

APCM: Apertura y cierre de mandíbula; APCL: Apertura y cierre de labios; PRLA: Protrusión y retracción de labios; PRLE: Protrusión y retracción de lengua; ML: Lengua a derecha e izquierda; MAD: Lengua en ascenso y descenso; Ref: Valores refereciales en población sin patología.

Por otra parte, con respecto a la articulación se observó una reducción en la producción de todas las sílabas medidas en una unidad de tiempo de 5 segundos. En cuanto la evaluación de diadicocinesias se observó un desempeño levemente más bajo en sujetos con EPOC en las distintas palabras medidas. Finamente, el número de palabras por minuto también presentó diferencia con los valores referenciales que fue menor en el caso de los pacientes con EPOC.

Tabla 4. Descriptivos de tarea de repetición, lectura y diadicocinesias

\begin{tabular}{|c|c|c|c|c|c|c|c|c|}
\hline \multicolumn{9}{|c|}{ Repetición } \\
\hline & \multicolumn{3}{|c|}{ Femenino } & \multicolumn{3}{|c|}{ Masculino } & \multicolumn{2}{|c|}{ Total } \\
\hline & $x / \sigma$ & Ref & $M e$ & $x / \sigma$ & Ref & $M e$ & $x / \sigma$ & $M e$ \\
\hline Repetición/pa/ & $12 \pm 2,91$ & 32,17 & 13 & $13,71 \pm 4,07$ & 34,05 & 11 & $12,60 \pm 3,36$ & 12,5 \\
\hline Repetición/ta/ & $13,38 \pm 3,12$ & 32,57 & 14 & $13,43 \pm 4,19$ & 33,66 & 13 & $13,40 \pm 3,4$ & 13 \\
\hline Repetición/ka/ & $10,92 \pm 3,68$ & 29,76 & 11 & $14,00 \pm 4,39$ & 30,91 & 12 & $12 \pm 4,11$ & 12 \\
\hline
\end{tabular}


Estado de los parámetros de habla en usuarios con enfermedad pulmonar obstructiva crónica (EPOC), reporte de una serie de casos

\begin{tabular}{|c|c|c|c|c|c|c|c|c|}
\hline \multicolumn{9}{|c|}{ Lectura } \\
\hline & \multicolumn{3}{|c|}{ Femenino } & \multicolumn{3}{|c|}{ Masculino } & \multicolumn{2}{|c|}{ Total } \\
\hline & $x / \sigma$ & Ref & Me & $x / \sigma$ & Ref & Me & $x / \sigma$ & Me \\
\hline PPM & $106 \pm 27,81$ & 159 & 123 & $116 \pm 22,8$ & 155 & 114,7 & $109 \pm 26,25$ & 119 \\
\hline \multicolumn{9}{|c|}{ Lectura } \\
\hline & \multicolumn{3}{|c|}{ Femenino } & \multicolumn{3}{|c|}{ Masculino } & \multicolumn{2}{|c|}{ Total } \\
\hline & $x / \sigma$ & Ref & Me & $x / \sigma$ & Ref & $M e$ & $x / \sigma$ & $M e$ \\
\hline /Pa-ta-ka/ & $9,08 \pm 1,75$ & 10,87 & 9 & $10,14 \pm 3,13$ & 12,52 & 10 & $9,45 \pm 2,3$ & 9 \\
\hline /Pitúko/ & $10,31 \pm 2,86$ & 11,98 & 10 & $10,14 \pm 2,93$ & 12,52 & 10 & $10,25 \pm 2,55$ & 10 \\
\hline /Petáka/ & $10,54 \pm 2,40$ & 11,79 & 11 & $11,57 \pm 4,42$ & 12,52 & 12 & $10,90 \pm 3,17$ & 11 \\
\hline /Botíka/ & $10,08 \pm 2,25$ & 11,68 & 10 & $11,14 \pm 3,53$ & 12,42 & 11 & $10,45 \pm 2,25$ & 10 \\
\hline /Bodéga/ & $10,23 \pm 1,87$ & 11,70 & 10 & $11,86 \pm 3,38$ & 12,43 & 11 & $10,80 \pm 2,54$ & 10,50 \\
\hline /Medíko/ & $10,38 \pm 2,29$ & 11,42 & 11 & $11,14 \pm 3,93$ & 11,85 & 10 & $10,65 \pm 2,88$ & 11 \\
\hline /Malága/ & $7,92 \pm 2,17$ & 9,49 & 8 & $9,43 \pm 4,07$ & 9,94 & 10 & $8,45 \pm 2,96$ & 8,5 \\
\hline Habla automática & $10,23 \pm 1,95$ & 9,23 & 10,34 & $10,46 \pm 1,63$ & 9,61 & 10,67 & $10,31 \pm 1,8$ & 10,45 \\
\hline
\end{tabular}

PPM: Palabras por minuto.Diadococinesias y repetición de palabras: número de repeticiones en 5 segundos. Habla automática: medida en segundos. Ref: Valores refereciales en población sin patología.

\section{DISCUSIÓN}

Los principales hallazgos del presente estudio fueron el deterioro en el TMF, control motor oral, número de palabras por minutos, diadicocinesias, lectura oral.

Si bien en la actualidad existen investigaciones en nuestro país que realizan estudios similares, estos han involucrado únicamente el estado de los parámetros de habla en adultos normales, por lo que este estudio aporta información relevante a considerar en el abordaje de esta patología, esto debido a que los parámetros del habla están asociado a la comunicación y con ello a la funcionalidad de las personas.

Los datos encontrados en nuestra investigación para el TMF del fonema $/ \mathrm{s} /$, considerando el sexo, fueron de 9,06 segundos en los hombres y 5,24 en las mujeres. Esto es notoriamente más bajo que los datos presentados por Toledo en 2011 que obtuvo 21,46 y 17,98 segundos respectivamente, en la misma prueba. Para los resultados obtenidos de la producción de /a/ estos fueron $6,08 \mathrm{~s}$ en mujeres y $7,87 \mathrm{~s}$ en hombres, mientras que los obtenidos por Toledo son $15,03 \mathrm{~s}$ y $16,30 \mathrm{~s}$, respectivamente. Lo mismo ocurre con la producción de /i/ donde se obtuvo $8,12 \mathrm{~s}$ en mujeres y $8,46 \mathrm{~s}$ en hombres mientras que la investigación utilizada como referencia se encontró 17,41 s y 20,13 s respectivamente ${ }^{(22)}$.

De acuerdo a los resultados de la investigación en la producción de / pa/ solicitada en 5 segundos Toledo obtuvo 32,17 repeticiones en mujeres y 34,05 repeticiones en hombres; nuestro estudio, en cambio, obtuvo un promedio de 12 repeticiones en pacientes de sexo femenino $y$
13,71 repeticiones en pacientes de sexo masculino. En la repetición de /ta/, Toledo entrega un resultado preliminar de normalidad de 32,57 y 33,66 repeticiones para mujeres y hombres, mientras que nuestro estudio muestra nuevamente una diferencia considerable con unas 13,38 y 13,43 repeticiones en mujeres y hombres, respectivamente. En relación a con la repetición de $/ \mathrm{ka} /$, 29,76 y 30,91 repeticiones son los datos de la investigación de referencia mientras que los obtenidos en nuestro estudio son de 10,92 y 14 repeticiones en hombres y mujeres en el mismo ítem, mostrando nuevamente una disminución en la realización del ejercicio ${ }^{(22)}$.

La diferencia entre los resultados obtenidos en los adultos con EPOC y los adultos normales disminuye en la realización de movimiento de protrusión y retrusión de labios solicitada en 5 segundos, presentándose similitud en los resultados. Toledo encuentra un promedio de 6,29 y 6,19 en mujeres y hombres respectivamente, mientras que en el presente estudio obtuvimos un promedio de 5 ciclos en mujeres y 7,29 ciclos en hombres. La diferencia se hace menor en el ejercicio solicitado de protrusión y retracción de la lengua en 5 segundos, donde Toledo obtuvo 5,27 y 6,07 ciclos en mujeres y hombres, mientras nuestra investigación entregó un resultado promedio de 5,92 y 7,71 ciclos en mujeres y hombres respectivamente, o en movimientos de la lengua de derecha a izquierda o arriba y abajo donde obtuvimos un total de 6,60 y 4,80 ciclos respectivamente mientras que Toledo encuentra un total de 8,06 y 5,87 ciclos en los mismos ejercicios.

Finalmente, para la repetición de la serie /pa-ta-ká/ obtuvimos un resultado en mujeres y hombres de 9,08 y 
10,14 repeticiones respectivamente, similar a lo obtenido por Toledo con 10,87 y 11,23 repeticiones en este ítem. Lo mismo ocurre con la repetición de las palabras /pi tú ko/, /pe tá ka/, /bo ti ka/, /bo dé ga/, /mé di ko/, /má la ga/ en los que los resultados presentados por Toledo estuvieron en un rango de 9,7-12,6, (cifras totales de lo realizado por hombres y mujeres), que presentan solo una variación menor con los resultados de nuestra investigación, en los que el rango fue de 8,45-10,90 repeticiones.

Es probable que la EPOC no influya de manera significativa en estas actividades debido a que investigaciones muestran que el enlentecimiento de estos movimientos estaría relacionado al avance de la edad ${ }^{(23)}$, lo que se puede comprobar al analizar los datos similares que se obtuvieron en los resultados del estudio de referencia realizado en adultos normales.

Dentro de los datos obtenidos en lectura, es necesario mencionar que en este ítem solo se consideraron 16 de los 20 pacientes de la muestra total. Esto se debe a que solo ese número de adultos sabían leer, pudiendo realizar dicha prueba. Los datos de nuestro estudio en tiempo de lectura corresponden a $62,06 \mathrm{~s}$ en mujeres con una cantidad de palabras por minuto de 106,74; y en hombres, un tiempo de 53,68 s y 116,16 palabras por minuto. Al observar los datos recabados por Toledo, encontramos que el tiempo de lectura de nuestro estudio era mayor al de los adultos normales (que obtenían un promedio de 39,6 s) y, por lo mismo, la cantidad de palabras por minuto era menor al de los adultos normales. En este caso, creemos que es importante considerar la variable de la afección que el EPOC podría tener en los pacientes, que puede ser la razón de una lectura más pausada; sin embargo, es también importante considerar la variabilidad que se presentó en los niveles de escolaridad de los participantes del estudio.

Por último, en los ítems de habla automática a velocidad normal y con velocidad aumentada se obtuvo un resultado de 10,23 y 5,98 segundos en mujeres para cada ítem, respectivamente. Mientras que los hombres realizaron el mismo ejercicio en 10,46 y 6,07 segundos respectivamente. Mostrando una pequeña variación con los resultados de Toledo en los que las mujeres obtuvieron 9,23 y $4,83 \mathrm{~s}$ mientras que los hombres realizaron la misma actividad en un tiempo de 9,61 y 4,60 segundos respectivamente.

La relevancia de mencionar estos datos reside en que ambos estudios comparten un rango etario similar, pese a esto, se presenta una variación considerable en los pacientes con EPOC, mostrando en los casos antes mencionados un resultado muy por debajo de los rangos de normalidad presentados por Toledo en el año $2011^{(22)}$. Ya que la enfermedad pulmonar obstructiva crónica se define como una obstrucción crónica al flujo aéreo ${ }^{(24)}$ y fueron en estas acciones donde se presenta el mayor descenso en nuestra investigación, se puede establecer una relación de la EPOC con la disminución de la capacidad de producir los fonemas de forma prolongada como se evalúan en el ítem de TMF.

Los resultados de esta investigación concuerdan con lo obtenido por Binazzi et al., en el 2011, quienes estudiaron la presencia de disnea durante el habla en usuarios con EPOC mediante la aplicación de un cuestionario, y concluyeron que los usuarios con EPOC reportan disnea durante tareas de conversación, alzar la voz, hablar en grupo, cantar, hablar en lugares ruidosos y llamar por teléfono ${ }^{(3)}$.

En relación a las limitaciones del estudio, es necesario indicar que no se consideraron subgrupos de estudio en función de la edad o el grado de severidad según los criterios GOLD, ya en relación a la alteración del volumen espirado en el primer segundo, índice BODE (de sus siglas en inglés body mass index, airflow obstruction, dysnea and excercise capacity index), o en base al fenotipo de EPOC que presentan los pacientes ${ }^{(25)}$, lo que permitiría realizar un análisis de tipo comparativo y correlacional entre nuevas variables de estudio; como grado de severidad y estado de los parámetros de habla; esto no se ejecutó debido a que al centro de salud sólo asistían pacientes GOLD 1 y GOLD 2. En cuanto al diseño investigación es posible mencionar que los estudios descriptivos no representan el mejor nivel de evidencia científica, por lo que se sugiere incorporar las variables a diseños de investigación con mayor control; también es importante mencionar que el nivel educativo puede sesgar la medición en tarea de lectura, por lo que se sugiere controlar esta variables o incorporar elementos de conversación espontánea a través de entrevista semiestructurada. otro de los elementos a incorporar es la medición de los parámetros acústicos del habla como la frecuencia fundamental, armónicos y índices de distorsión de la frecuencia, como medidas más sensibles para valorar y cuantificar el impacto de la patología sobre el habla.

Los resultados de esta investigación permiten dar a conocer una primera aproximación desde la descripción de los parámetros del habla, ademas, abre la posibilidad de indagar en estos aspectos en futuras investigaciones, es por ello que parece pertinente considerar la necesidad de continuar investigaciones tendiente a valorar los aspectos de la voz, el habla y comunicación, con miras a mejorar la práctica clínica y la calidad de vida en con EPOC ya que esta no es valorada habitualmente en cuestionarios o escalas de funcionalidad en actividades de la vida diaria (26). Finalmente, consideramos que el habla debe ser estudiada en los usuarios con EPOC, ya que limitaciones en la comunicación verbal afectan la calidad de vida e impactan en atención integral del usuario dentro de la práctica clínica cotidiana. 


\section{REFERENCIAS BIBLIOGRÁFICAS}

1. Mathers C, Loncar D. Projections of Global Mortality and Burden of Disease from 2002 to 2030. PLoS Med. 2006; 3(11): e442.

2. Organización Mundial de la Salud [sede Web]. Enfermedades Respiratorias Crónicas. Departamento de Enfermedades Crónicas y Promoción de la Salud; Ginebra: OMS; 2016. Disponible en: www.who.int/respiratory/copd/es/

3. Binazzi B, Lanini B, Romagnoli I, Garuglieri S, Stendardi $\mathrm{L}$, Bianchi $\mathrm{R}$ et al. Dyspnea during Speech in Chronic Obstructive Pulmonary Disease Patients: Effects of Pulmonary Rehabilitation. Respiration. 2011; 81(5): 379-385.

4. Ministerio de Salud. Guía Clínica AUGE: Enfermedad Pulmonar Obstructiva Crónica (EPOC): Santiago: MINSAL, Sub Secretaría de Salud Pública; 2013.

5. García-Río F, Lores V, Rojo B. Evaluación Funcional Respiratoria (obstrucción y atrapamiento). Arch Bronconeumol. 2007; 43(3): 8-14.

6. González RA, Bevilacqua JA. Las disartrias. Rev Hosp Clín Univ Chile. 2012; 23: 299-309

7. Binazzi B, Lanini B, Bianchi R, Romagnoli I, Nerini M, Gigliotti $\mathrm{F}$, et al. Breathing pattern and kinematics in normal subjects during speech, singing and loud whispering. Acta physiologica. 2006; 186(3): 233-246.

8. Hyatt RE. The Interrelationships of Pressure, Flow, and Volume during Various Respiratory Maneuvers in Normal and Emphysematous Subjects. Am Rev Respir Dis.1961; 83(5): 676 683.

9. O’Donnell DE, Sanii R, Anthonisen NR, Younes M. Effect of dynamic airway compression on breathing pattern and respiratory sensation in severe chronic obstructive pulmonary disease. Am Rev Respir Dis. 1987;135(4):912-918.

10. Eltayara L, Becklake MR, Volta CA, Milic-Emili J. Relationship between chronic dyspnea and expiratory flow limitation in patients with COPD. Am J Respir Crit Care Med 1996;154(6 Pt 1):1726-1734

11. Kent RD, Kent JF, Rosenbek JC. Maximum Performance Test of Speech Production. J Speech Hear Res. 1987; 52(4):367-387.

12. Kent RD. Nonspeech Oral Movements and Oral Motor Disorders: A Narrative Review. Am J Speech Lang Pathol. 2015; 24(4): 763-789.

13. Gutiérrez S, Domínguez A, Valenzuela A. Eficacia de los criterios clínicos y factores de riesgo en el diagnóstico de enfermedad pulmonar obstructiva crónica. Med Int Méx. 2014; 30(3): 247-256.

14. Carrillo R, Flores O, Zepeda A, Maldonado R, Meza J. Curva flujo/volumen en paciente con enfermedad pulmonar obstructiva crónica exacerbada. Rev Asoc Mex Med Crit y Ter Int. 2015; 29(4): 244-246

15. Oyarzún $M$. ¿Qué se investiga en relación a la enfermedad pulmonar obstructiva crónica en Chile?. Rev Chil Enf Respir. 2002; 18(3): 189-198

16. Blanco-Aparicio M, Vázquez Rodríguez I, Verea-Hernando $\mathrm{H}$. Adaptación trascultural al español del Airways Questionnaire 20 (AQ20), un cuestionario de calidad de vida abreviado para la evaluación clínica del asma y la EPOC. Arch Bronconeumol. 2009; 45(1): 24-29

17. Vinaccia S, Riveros F, Quiceno J. Análisis exploratorio del Airways Questionnaire 20 (AQ20) en pacientes colombianos con enfermedad pulmonar obstructiva crónica. Rev Am Med Respir. 2017; 17(1): 83-89.

18. Hernández T. Elías D. González Vergara. Calidad de vida aplicada

a la Neumología. En: Soto JG Manual de diagnóstico y terapéutica en neumología. 2a ed. Madrid: Ergon;2004. 113-123.

19. Manterola C, Otzen T. Estudios Observacionales: Los Diseños Utilizados con Mayor Frecuencia en Investigación Clínica. Int J Morphol. 2014;32(2): 634-645.

20. Quiroga P, Albala C, Klaasen G. Validación de un test de tamizaje para el diagnóstico de demencia asociada a edad, en Chile. Rev Méd Chile. 2004; 132: 467-478

21. Bahamonde C, González J, Martínez M, Muñoz M. Estandarización de parámetros cuantificables de habla en adultos normales Chilenos. Seminario de Investigación, Escuela de Fonoaudiología, Universidad de Chile, 2007.

22. Toledo L, Bahamonde D, González J, Martínez M, Muñoz M, Muñoz D. Parámetros del habla en adultos normales chilenos. Rev Chil de Fonoaudiología. 2011; 10: 33-43

23. Bilodeau-Mercure M., Kirouac V, Langlois N, Ouellet C, Gasse I. Tremblay, P. Movement sequencing in normal aging: speech, orofacial, and finger movements. Age (Dordr).2015; 37(4): 78.

24. Gutiérrez S, Domínguez A, Valenzuela A. Eficacia de los criterios clínicos y factores de riesgo en el diagnóstico de enfermedad pulmonar obstructiva crónica. Med Int Méx. 2014; 30(3): 247-256.

25. Golpe P, Sanjuán P, Cano E, Castro O, Pérez L. Distribución de fenotipos clínicos en pacientes con enfermedad pulmonar obstructiva crónica por humo de biomasa y por tabaco. Arch Bronconeumol. 2014; 50(8): 318-324.

26. Hodgev V, Kostianev S, Marinov B. University of Cincinnati Dyspnea Questionnaire for Evaluation of Dyspnoea during physical and speech activities in patients with chronic obstructive pulmonary disease: a validation analysis. Clin Physiol Funct Imaging. 2003; 23(5): 269-274.

Fuentes de financiamiento:

Este artículo ha sido financiado por los autores.

Conflictos de interés:

Los autores declaran no tener ningún conflicto de interés.

\section{Correspondencia:}

Carla Soledad Figueroa Saavedra

Dirección: Universidad Autónoma de Chile Avenida Pedro de Valdivia 425, Providencia. Santiago, Chile.

Teléfono: +(56) (2) 23036000

Correo electrónico: carlafig@gmail.com

\section{Recibido: 16 de mayo de 2018 \\ Evaluado: 01 de junio de 2018 Aprobado: 10 de agosto de 2018}

(c) La revista. Publicado por Universidad de San Martín de Porres, Perú. (cc) $\mathbf{B r}$ Licencia de Creative Commons Artículo en acceso abierto bajo términos de Licencia Creative Commons Atribución 4.0 Internacional. (http://creativecommons.org/licenses/by/4.0/)

\section{ORCID iDs}

\section{Joaquín Gay}

Atalia Catrileo

Evanely Sepulveda

Carla Soledad Figueroa Saavedra

Sandra Barria

Daniela Arredondo

María Paz Moya https://orcid.org/0000-0003-1687-5971 https://orcid.org/0000-0002-0191-6446 https://orcid.org/0000-0003-2480-1016 https://orcid.org/0000-0002-4184-8951 https://orcid.org/0000-0001-6686-9073 https://orcid.org/0000-0002-4070-6871 https://orcid.org/0000-0003-2889-3667 